\title{
Magnetic recykling of complex catalysts immobilized on thiol-functionalized polymer supports
}

\author{
Natalia Bączek*, Krzysztof Strzelec, Karolina Wąsikowska \\ Lodz University of Technology, Institute of Polymer and Dye Technology, Faculty of Chemistry, ul. Stefanowskiego 12/16, \\ 90-924 Lodz, Poland \\ *Corresponding author: e-mail: natalia.baczek@interia.pl
}

\begin{abstract}
In this work, the application of the thiol-functionalized epoxy resin encapsulated on magnetic core as supports for palladium catalysts is reported. The study focuses on obtaining of heterogeneous catalysts which can be separated by magnetic field. Palladium complex catalyst $\left[\mathrm{PdCl}_{2}(\mathrm{PhCN})_{2}\right]$ has been heterogenized by anchoring to these supports via ligand exchange reaction. The characterization of polymeric supports and heterogenized palladium catalysts has involved research methods like time-of-flight secondary ion mass spectrometry (TOF-SIMS), scanning electron microscopy (SEM) and nitrogen BET surface area measurements. The activity and stability during long-term use of the investigated catalytic systems were tested in a Heck and hydrogenation reaction. The influence of the type of thiols used as epoxy hardeners and the morphology of the supports on the catalytic properties of epoxy-supported palladium catalysts was discussed.
\end{abstract}

Keywords: encapsulation, magnetic recycling, epoxy resin, supported catalysts, Heck reaction, hydrogenation reaction.

\section{INTRODUCTION}

Encapsulation is a process in which some particles are surrounded by a coating to give small capsules with many useful properties. The material inside the microcapsule is referred to as a core, internal phase, or fill, whereas the wall is sometimes called a shell, coating, or membrane. The technique of encapsulation depends on the physical and chemical properties of the material to be encapsulated. In the literature there are many reports on the encapsulation process, however the list of their application in catalysis is rather small. Supported salen-metal complexes prepared with polyethylene ligands (PEOlig) that have utility in catalysis also have enhanced stability toward acid. PEOlig -bound $\mathrm{Cr}(\mathrm{III})$-salen and $\mathrm{Mn}(\mathrm{III})$-salen complexes suspended in a non-swelling solvent such as methanol do not react with trifluoroacetic acid in methanol. The stability of these metal complexes shows that PE ligands and a PE matrix can have additional utility in recycling catalysts in that the solid state environment of the recovered species can minimize adventitious reactions that decompose a catalyst during catalyst recycling ${ }^{1}$. The immobilization of metal nanoparticles in magnetic responsive solids allows the easy, fast, and clean separation of catalysts. The efficiency of this separation process depends on a strong metal-support interaction. This interaction can be enhanced by functionalizing the support surface with amino groups. The catalyst supports contain a magnetic core which is able to the magnetic separation from liquid systems and an external surface of silica suitable for further modification with organosilanes. Jacinto prepared a magnetically recoverable amino-functionalized support captured iridium species from liquid solutions and produced a highly active hydrogenation catalyst with irrelevant metal leaching. An analogous $\operatorname{Ir}^{0}$ catalyst prepared with use of a nonfunctionalized support shows a higher degree of metal leaching into the liquid products. The catalytic performance in the hydrogenation of alkenes is compared with that of $\mathrm{Rh}$ and Pt catalysts ${ }^{2}$.
Nowadays epoxy systems have various applications as coating materials, adhesives and composites and have a dominant position compared to other polymers. Epoxy carriers for complex catalyst are obtained by modification of epoxy networks by introduction of metal complexes into the structure of the cured matrix. There have been only a few reports on the use of epoxy resins as carriers of metal complex catalysts in organic synthesis. Epoxy resins doped with $\mathrm{Mo}, \mathrm{Pd}$, and $\mathrm{Rh}$ complexes have high catalytic stability in the processes of hydrogenation and hydroformylation.

In our earlier works we proposed similar approach and used bisphenol epoxy resin cured with ammonium ionic liquids as support for palladium complex catalyst. The usage of ionic liquids as hardeners for epoxy resins could greatly affect the activity of the catalyst without necessity of additional functionalization to improve of ligand-binding affinity to metal complexes. Investigated catalysts showed good selectivity during prolonged use in the Heck reaction ${ }^{3}$.

In the present study, we describe our approach to prepare the ferromagnetic catalysts by immobilizing the palladium complex on the functionalized epoxy resin. These catalysts were evaluated for their performance in the Heck and hydrogenation reaction.

\section{EXPERIMENTAL}

\section{Materials}

All organic reagents used in this study were supplied from Sigma-Aldrich and were used as received without further purification. Epoxy resin bisphenol A epichlorohydrin medium molecular weight $<700$ Epidian 5 (EP5), epoxy equivalent 200, (viscosity 20000-30000 $\mathrm{mPas}$ at $23^{\circ} \mathrm{C}$ ), and triethylenetetramine (Z-1), Organika Sarzyna, Poland were used. Iodobenzene (Aldrich), triethylamine (Organika Sarzyna), methyl acrylate (Aldrich) and N-metylhylpyrrolidone (ABCR) were used 
for Heck reaction. Cinamaldehyde (Aldrich) was used for hydrogenation reaction.

\section{Preparation of the carriers}

The carrier was prepared using encapsulation technique of ferromagnetic macroparticles by thiol functionalized epoxy resin. The iron filings with an average particle size of $2.3 \mathrm{~mm}$ were used as ferromagnetic cores. In these studies the multifunctional thiols: 3,6-dioxa-1,8-octanedithiol (DODT), trimethylopropane tris(3-mercaptopropionate) (TTMA), pentaerythritol tetrakis(3-mercaptopropionate) (PETMP) were applied to the functionalization of epoxy resin. The spectroscopy characterization and chemical structure of the epoxy resins cured with DODT, TTMA and PETMP were presented in our earlier papers ${ }^{4}$.

$5 \mathrm{~g}$ of iron filings with $150 \mathrm{ml}$ of acetone were placed in a two-necked flask equipped with a mechanical stirrer. Then the first portion of previously prepared mixture of an epoxy resin dissolved in a small amount of acetone, curing agent Z-1 and thiols (DODT, TTMA or PETMP) was added and stirred for $5 \mathrm{~h}$ at $55^{\circ} \mathrm{C}$. After the first cycle of $5 \mathrm{~h}$ the mixture was allowed to cool at room temperature. In the next step, after cooling, another portion of the mixture (EP5 + Z-1 + DODT, TTMA, PETMP) was added to the flask and was mixed for next $5 \mathrm{~h}$ at $55^{\circ} \mathrm{C}$. After this time, $50 \mathrm{ml}$ of ethanol was added to the solution and the mixture was allowed to cool to room temperature. Then, the solvent was evaporated under vacuum at $80^{\circ} \mathrm{C}$. Prepared carrier was transferred to a Petri dish and dried in an oven at $80^{\circ} \mathrm{C}$ for about $15 \mathrm{~min}$.

\section{Catalyst preparation}

A typical procedure was followed. To a known amount of the support $(0.3 \mathrm{~g})$ in a round-bottom flask, $\mathrm{PdCl}_{2}(\mathrm{PhCN})_{2}$ dissolved in $10 \mathrm{ml}$ of toluene was added. The mixture was stirred at room temperature for 2-3 days when all Pd complex had reacted with the polymer. The yellow product was filtrated off, extracted with toluene under nitrogen in order to remove complex that was non-chemically bound to the polymer and finally dried under vacuum. For all supported catalysts the Pd content was fixed below the metal uptake capacity for a polymer. The dry catalysts could be stored in air for many months without change.

\section{Morphology studies}

The morphology of samples was investigated with a scanning electron microscope JEOL 5500 LV, working in high vacuum and accelerating voltage of $10 \mathrm{kV}$.

Pore size distribution parameters were determined by application of the BET method on Sorptomatic 1900 FISONS Instrument.

\section{Catalyst test}

The activity and stability of the palladium catalysts was tested during a multiple use in the model Heck and cinnamaldehyde hydrogenation reaction.

The hydrogenation reactions were carried out in $100 \mathrm{ml}$ Parr microreactor model 4593 with glass liner. The reactor was charged with $0.2 \mathrm{~g}$ of supported palladium catalyst, $5 \mathrm{ml}$ of $96 \%$ ethanol, $0.25 \mathrm{ml}$ of toluene and $0.25 \mathrm{ml}$ of cinnamaldehyde. The reactor was flushed several times with pure hydrogen and then the hydrogen pressure and temperature were adjusted to the required level, 50 bar and $80^{\circ} \mathrm{C}$, respectively.

The typical Heck reaction was carried out in $50 \mathrm{ml}$ laboratory reactor. The reactor was charged with $0.05 \mathrm{~g}$ of supported palladium catalyst, $0.28 \mathrm{ml}(2.5 \mathrm{mmol})$ of iodobenzene, $0.23 \mathrm{ml}(2.5 \mathrm{mmol})$ of methyl acrylate, $0.15 \mathrm{ml}(1.4 \mathrm{mmol})$ of toluene, $0.35 \mathrm{ml}(2.5 \mathrm{mmol})$ of triethylamine and $1.00 \mathrm{ml}(10.4 \mathrm{mmol})$ of N-methylpyrrolidone (NMP). The reaction was conducted at a temperature of $80^{\circ} \mathrm{C}$ for 1.5 hours. Samples were taken every 5, 15, 30, 60 and 90 minutes.

For both reactions, the reaction products were quantitatively analyzed by gas chromatography (GC) on a Hewlett-Packard 5990 II gas chromatograph equipped with a thermal conductivity detector. The GC column was HP-50+ (crosslinked 50\% Ph Me silicone) $30 \mathrm{~m}$ x $0.63 \mathrm{~mm} \times 1.0 \mu \mathrm{m}$ film thickness. The product was identified by matching retention times with those of authentic samples.

In the recycling tests, when reaction was completed, the catalyst was separated by magnetic field, washed with $3 \times 25 \mathrm{ml}$ of toluene, vacuum dried and used for the next reaction.

\section{RESULTS AND DISCUSSION}

\section{Synthesis and characterization of palladium catalysts}

By the incorporation of the thiol groups to the epoxy matrix we received a polymer chain with ligands able to chelating the palladium complex. This procedure leads to a controlled introduction of bonding groups to the polymeric structure. The metal complex was introduced to the matrix via ligand exchange reaction.

The support microporous structure can influence the selectivity of reaction at a given site. The steric demands of a catalytic centre bound to a support can be very different to those of its homogeneous analogue. Microporous structure of the support affects the accessibility of the interior of the swollen resin and so enhance the selectivity by increasing diffusional restrictions for reaction species. Therefore, we were particularly interested to learn about the porous structure of these materials in the range of micropores $(<2 \mathrm{~nm})$ and mesopores $(>2 \mathrm{~nm}, 50<\mathrm{nm})$, being of the same order of dimension as those of reacting molecules.

The results determined by nitrogen BET adsorption method (Table 1) indicate that investigated supports have different pore size distribution which probably arise from differences in their crosslink densities. The catalyst showed disparate pore size distribution with the micropores (radii below $2 \mathrm{~nm}$ ) and very low volume of meso- and macropores.

The PETMP-cured resin exhibits the lower value of average pore size, the lower surface area and lower porosity than epoxy resin/DODT and epoxy resin/TTMA-based supports.

For all supports immobilization of palladium precursor resulted in a similar loss in surface area and corresponding decrease in mean pore diameter. The differences in porous structure of these supports were also confirmed by characterization of surface morphology by scanning 
Table 1. BET Data for the supports and supported Pd catalysts

\begin{tabular}{|l|c|c|}
\hline $\begin{array}{l}\text { Polymer support or } \\
\text { supported Pd catalyst }\end{array}$ & $\begin{array}{c}\mathrm{N}_{2} \text { surface } \\
\text { area } \\
{\left[\mathrm{m}^{2} / \mathrm{g}\right]}\end{array}$ & $\begin{array}{c}\text { Average pore } \\
\text { diameter } \\
{[\mathrm{nm}]}\end{array}$ \\
\hline Epoxy resin/DODT & 89 & 23 \\
Epoxy resin/DODT /Pd & 82 & 20 \\
Epoxy resin/TTMA & 78 & 21 \\
Epoxy resin/TTMA/Pd & 76 & 18 \\
Epoxy resin/PETMP & 54 & 6 \\
Epoxy resin/PETMP/Pd & 51 & 4 \\
\hline
\end{tabular}

electron microscopy SEM. The noticeable different microporous structure of the DODT and TTMA-cured resins presented in Fig. 1, can influence the activity of
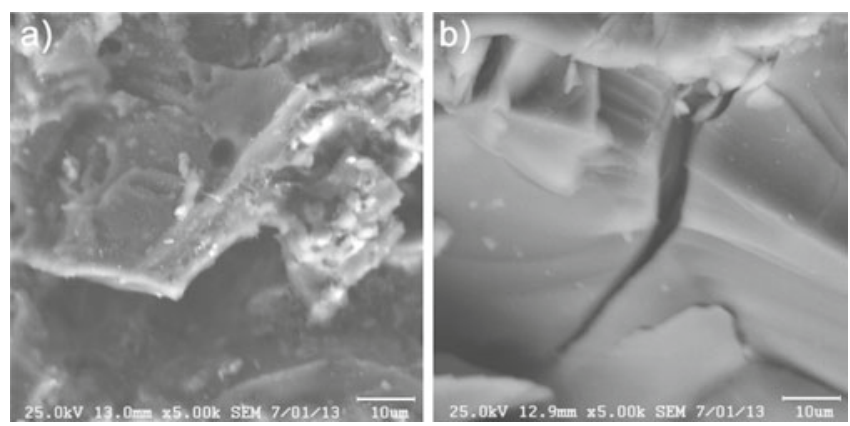

Figure 1. SEM micrographs a) epoxy resin/DODT; b) epoxy resin/TTMA

catalyst by the steric demands of a catalytic centre bound to these supports.

\section{Activity and stability tests}

The effect of the structure of ferromagnetic polymer support on the stability, catalyst activity and the selectivity was examined in the Heck reaction and hydrogenation of cinnamaldehyde. The selective hydrogenation of $\alpha, \beta-$ -unsaturated carbonyl compounds to their corresponding unsaturated alcohols is an important step in the preparation of various fine chemicals such as fragrances for the perfume industry. The catalytic hydrogenation of $\alpha, \beta$-unsaturated aldehydes to produce unsaturated alcohols is a difficult challenge because the hydrogenation of an alkene double bond catalyzed by noble metals is thermodynamically and kinetically more favored over the hydrogenation of a carbonyl group. The hydrogenation<smiles>O=C/C=C/c1ccccc1</smiles>

Cinnamaldehyde<smiles>O=CCCc1ccccc1</smiles>

Hydrocinnamylaldehyde (HCALD) (CALD)

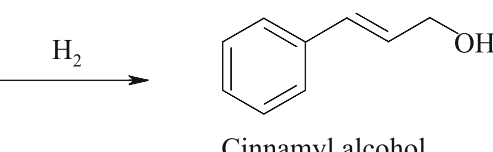

Cinnamyl alcohol (CALC)

$\mathrm{H}_{2}$ $\checkmark$

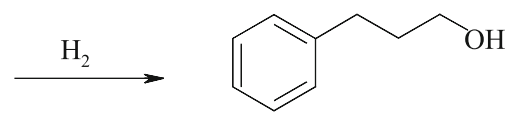

Hydrocinnamyl alcohol (HCALC)
Scheme 1. Hydrogenation of cinnamaldehyde of cinnamaldehyde can give rise to three products, namely hydrocinnamaldehyde (HCALD), cinnamyl alcohol (CALC), and hydrocinnamyl alcohol (Scheme 1).

Table 2 lists the results of the liquid phase hydrogenations of cinnamaldehyde at 50 bar pressure and $80^{\circ} \mathrm{C}$. For all tested catalytic systems, supported palladium complex $\mathrm{PdCl}_{2}(\mathrm{PhCN})_{2}$ catalysts have been found to give poor selectivity to cinnamyl alcohol (CALC) after subsequent use, due to the reduction of the supported precursor. Cinnamyl alcohol (CALC) is strongly adsorbed on the $\operatorname{Pd}(0)$ catalyst surface and may undergo isomerisation to the hydrocinnamaldehyde (HCALD). The catalyst selectivity seems also depend on the size distribution of the metal nanoparticles generated during the first reaction cycle. The growth of the metal nanoparticles during reduction becomes limited by the steric restrictions imposed by support nanoscale morphology. Different metal particle size distribution influenced by morphology of the support can be an explanation for the unequal catalyst performances of the epoxy resin/ PETMP/Pd catalyst, with the smallest size of nanopores. Larger palladium nanoparticles created on epoxy resin/ DODT/Pd and epoxy resin/DODT/Pd systems with a larger pore size, were found to give higher selectivity to cinnamyl alcohol (CALC). The quite low selectivities of cinnamyl alcohol were reported for $\mathrm{Ru} / \mathrm{Al}_{2} \mathrm{O}_{3}$ catalyst $(30 \%)^{5}$. No cinnamyl alcohol was detected as a product of reaction conducted over silica supported Pd catalysts ${ }^{6}$. Also, supported iridium, cobalt, osmium, and platinum catalysts have been found to give poor selectivity to unsaturated alcohols, but high selectivity can be induced by the addition of small amounts of metal chlorides such as $\mathrm{FeCl}_{2}{ }^{7}$.

Table 2. The hydrogenation of cinnamaldehyde over the epoxy resin/DODT/Pd, epoxy resin/TTMA /Pd and epoxy resin/PETMP/Pd

\begin{tabular}{|l|c|c|c|c|c|}
\hline $\begin{array}{l}\text { Ferromagnetic } \\
\text { Catalyst }\end{array}$ & Run & $\begin{array}{c}\text { Conversion } \\
{[\%]}\end{array}$ & $\begin{array}{c}\text { HCALD } \\
{[\%]}\end{array}$ & $\begin{array}{c}\text { HCALC } \\
{[\%]}\end{array}$ & $\begin{array}{c}\text { CALC } \\
{[\%]}\end{array}$ \\
\hline \multirow{2}{*}{ Epoxy resin/DODT /Pd } & 1 & 98 & 10 & 67 & 23 \\
\cline { 2 - 6 } & 3 & 96 & 2 & 96 & 2 \\
\hline \multirow{2}{*}{ Epoxy resin/TTMA /Pd } & 1 & 97 & 8 & 60 & 32 \\
\cline { 2 - 6 } $\begin{array}{l}\text { Epoxy } \\
\text { resin/PETMP/Pd }\end{array}$ & 3 & 96 & 2 & 97 & 1 \\
\cline { 2 - 6 } & 3 & 97 & 7 & 63 & 30 \\
\hline
\end{tabular}

The stability of the supported catalyst was tested during repeated catalysis runs. Recycling the immobilized palladium catalysts three times demonstrated their high stability despite the changes that occur in their structure during the catalytic reaction.

The activity and stability of the palladium catalysts were also researched during their multiple usage in the model Heck reaction. The Heck reaction coupling iodobenzene with methyl acrylate, occurring in the solution of N-methylpyrrolidone (NMP) in the presence of triethylamine. The only observed product was methyl trans-cinnamate (Scheme 2).

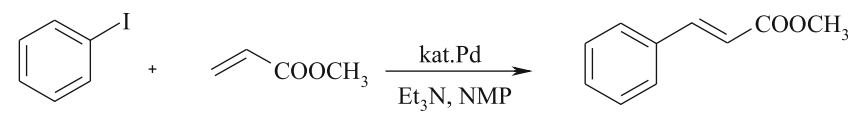

iodobenzene methyl acrylate methyl trans-cinnamate

Scheme 2. Heck reaction 
The activity of all prepared catalyst was similar to homogeneous palladium precursor $\mathrm{PdCl}_{2}(\mathrm{PhCN})_{2}$ (Table 3 ). The lower catalytic activity of the catalysts supported on the epoxy resin/PETMP we ascribe to fewer Pd sites being accessible to the reactants when these are attached to the polymer of lower microporosity. The better availability of the catalytic sites situated in a DODT and TTMA cured resins having a greater volume of micropores and higher surface area, increases the activity of supported Pd systems. The activities of all tested catalysts decrease with the subsequent usage which might be caused by the palladium leaching. In order to monitor the deactivation process time-of-flight secondary ion mass spectrometry (TOF-SIMS) was used. TOF-SIMS method permits the evaluation of the chemical composition of the catalyst surface, the nature of interactions between metallic phases and supports as well as the distribution of metal or other catalyst components on the surface. However, the quantitative analysis by TOF-SIMS technique is relatively difficult. TOF-SIMS may be very useful in the studies of catalyst deactivation processes. Catalysts after 8th use were analyzed and compared to the fresh samples. A small drop of the amount of surface accessible palladium was observed however the drop of Pd content was at a safe level and did not significantly affect the activity.

Table 3. The Heck reaction

\begin{tabular}{|l|c|c|c|}
\hline Catalyst & Run & $\begin{array}{c}\text { Conversion } \\
{[\%]}\end{array}$ & $\begin{array}{c}\text { Intensity of } \\
{ }^{106} \mathrm{Pd}^{+}\end{array}$ \\
\hline Homogeneous & 1 & 98 & - \\
\hline $\mathrm{PdCl}_{2}(\mathrm{PhCN})_{2}$ & 1 & 96 & $7.2 \times 10^{-3}$ \\
\hline Epoxy resin/DODT/Pd & 4 & 93 & - \\
& 8 & 90 & $5.1 \times 10^{-3}$ \\
\hline \multirow{3}{*}{ Epoxy resin/TTMA/Pd } & 1 & 84 & $6.3 \times 10^{-3}$ \\
& 4 & 77 & - \\
\hline \multirow{2}{*}{ Epoxy resin/PETMP/Pd } & 8 & 75 & $4.4 \times 10^{-3}$ \\
& 4 & 80 & $4.3 \times 10^{-3}$ \\
& 8 & 79 & - \\
\hline
\end{tabular}

Reaction conditions: temperature $80^{\circ} \mathrm{C}$; substrates: iodobenzene $0.28 \mathrm{ml}(2.5 \mathrm{mmol})$, methyl acrylate $0.23 \mathrm{ml}(2.5 \mathrm{mmol})$; solvent: toluene $0.15 \mathrm{ml}(1.4 \mathrm{mmol})$, triethylamine $0.35 \mathrm{ml}(2.5 \mathrm{mmol})$, $\mathrm{N}$-methylpyrrolidone (NMP) $1.00 \mathrm{ml}(10.4 \mathrm{mmol})$; catalyst: $0.05 \mathrm{~g}$ (Pd content $-0.07 \mathrm{mM} / \mathrm{g}$ ); the conversion refers to $\mathrm{GC}$ analysis. ${ }^{*}$ Conversion of iodobenzene after $1.5 \mathrm{~h}$.

\section{CONCLUSIONS}

The results demonstrated that the prepared supports meet the requirements for organic supports in that they can be readily synthesized in a form suitable for heterogenization of metal complex catalysts and do not need any further functionalization. It was shown that thiol-functionalized epoxy resins can be used as coating for magnetic core in encapsulation process. This novel type of supports for metal complex catalysts can be easy separated by the use of magnetic field. Our experiments have shown that the microporous structure of the polymer supports in the hydrogenation of cinnamaldehyde seems to have higher impact on the reaction activity and selectivity as compared with the influence of the chemical structure of the supports. The morphology parameters such as pore size distribution and specific surface area depends on the type of thiol used as resin hardener.

The support morphology can also affect the catalytic properties of the epoxide-supported palladium catalyst in the Heck reaction. The better availability of the catalytic sites situated in resins having a greater volume of micropores and higher surface area, increases the activity of supported Pd systems. On the basis of recycling efficiency tests, it was found that the all epoxy-supported palladium catalysts have a high catalytic stability for eight consecutive runs. Future work will be devoted to the application of these catalysts for other reactions and characterization of the mode of bonding between the metal atom and the functional groups of the thiolmodified resin.

\section{ACKNOWLEDGEMENT}

This work was supported by the National Science Centre (Poland) (grant N ${ }^{\circ}$ 0255/B/H03/2011/40).

\section{LITERATURE CITED}

1. Suriboot, J., Hobbs, C.E., Yang, Y. \& Bergbreiter, D.E. (2012). Protective Encapsulation of acid-sensitive catalysts using polyethylene ligands. J. Polym. Sci., 50, 4840-4846. DOI: 10.1002/pola.26319.

2. Jacinto, M.J., Silva, F.P., Kiyohara, P.K., Landers, R. \& Rossi, L.M. (2012). Catalyst recovery and recycling facilitated by magnetic separation: iridium and other metal nanoparticles. ChemCatChem., 4, 698-703. DOI: 10.1002/cctc.201100415.

3. Ostrowska, S., Markiewicz, B., Wąsikowska, K., Bączek, N., Pernak, J. \& Strzelec, K. (2012). Epoxy resins cured with ionic liquids as novel supports for metal complex catalysts. C.R. Chimie, DOI: 10.1016/j.crci.2012.12.005.

4. Strzelec, K., Bączek, N., Ostrowska, S., Wąsikowska, K., Szynkowska, M.I. \& Grams, J. (2012). Synthesis and characterization of novel polythiourethane hardeners for epoxy resins. C.R. Chimie, 15, 1065-1071. DOI: 10.1016/j.crci.2012.09.003.

5. Lashdaf, M., Krause, A.O.I., Lindblad, M., Tiitta, M. \& Venalainen, T. (2003). Deposition of palladium and ruthenium $\beta$-diketonates on alumina and silica supports in gas and liquid phase. Appl. Catal. A., 241, 51-63.

6. Cairns, G.R., Cross, R.J. \& Stirling, D. (1997). Hydrogenation of cinnamaldehyde using catalysts prepared from supported palladium phosphine complexes. J. Catal., 166, 89-97 (9).

7. Chambers, A., Jackson, S.D., Stirling, D. \& Webb, G. (1997). Selective Hydrogenation of Cinnamaldehyde over Supported Copper Catalysts. J. Catal., 168, 301-314. 